Check for updates

Cite this: RSC Adv., 2018, 8, 17967

Received 10th April 2018

Accepted 1st May 2018

DOI: $10.1039 / \mathrm{c} 8 \mathrm{ra0} 3061 \mathrm{~h}$

rsc.li/rsc-advances

\section{Efficient preparation of crack-free, low-density and transparent polymethylsilsesquioxane aerogels via ambient pressure drying and surface modification}

\author{
Tiemin Li, Ai Du, D* Ting Zhang, Wenhui Ding, Mingfang Liu, Jun Shen, Zhihua Zhang \\ and Bin Zhou*
}

Polymethylsilsesquioxane (PMSQ) aerogels have gained considerable attention due to their high transparency, good mechanical properties and low thermal conductivity. However, low-density PMSQ aerogels are difficult to obtain by ambient pressure drying due to irreversible shrinkage. Inspired by previous research, we speculate that reducing surface silanol groups could reduce irreversible shrinkage along with the skeleton-strengthening effect. In addition, extending the ageing process is expected to lead to increased density. Thus, in this paper, we applied a mature technique to modify the surfaces of PMSQ gels with terminal silane groups to reduce hydrophilic surface silanol groups without strengthening the skeletons. This surface modification process greatly reduced irreversible shrinkage and allowed the PMSQ gels to return to their original sizes, in accompany with the decrease of silanol group (NMR results as the direct evidence). This method exhibits extremely high efficiency in the preparation of crack-free, low-density and transparent PMSQ aerogels. The PMSQ aerogels dried at ambient pressure had a low density of $48 \mathrm{mg} \mathrm{cm}^{-3}$, low thermal conductivity $\left(21.1 \mathrm{~mW} \mathrm{~m}^{-1} \mathrm{~K}^{-1}\right)$, high transparency $(81.3 \%$ at $550 \mathrm{~nm}$ ), super-hydrophobicity (contact angle of $155^{\circ}$ ) and excellent mechanical properties. The proposed method will be useful for the industrial production of transparent insulating materials and has potential applications in space exploration.

\section{Introduction}

Aerogels are porous materials obtained via sol-gel processes and supercritical drying. ${ }^{1-5}$ Among them, silica aerogels are the most studied due to their unique properties such as high specific surface area (500-1200 $\left.\mathrm{m}^{2} \mathrm{~g}^{-1}\right)$, high porosity (80$99.8 \%)$, low density $\left(\sim 0.003 \mathrm{~g} \mathrm{~cm}^{-3}\right)$, low thermal conductivity (0.005 $\left.\mathrm{W} \mathrm{m}^{-1} \mathrm{~K}^{-1}\right)$, ultra-low dielectric constant $(k=1.0-2.0)$, high visible-light transmittance and low refractive index $(\sim 1.05) .^{6-12}$

However, the friability of silica aerogels, which results from the weak connection of its network, and the need for supercritical drying limit the possibility of industrial production. PMSQ aerogels were invented by Kanamori et al. ${ }^{13}$ These aerogels show improved mechanical properties such as high compressibility, low thermal conductivity and fine transparency. ${ }^{13-17}$ Aerogel-like xerogels with no significant difference in bulk density, visible-light transmittance and thermal conductivity can be obtained by ambient pressure drying. ${ }^{\mathbf{1 3 , 1 4 , 1 8 , 1 9}}$ People commonly think that the ambient

Shanghai Key Laboratory of Special Artificial Microstructure Materials and Technology, School of Physics Science and Engineering of Tongji University, No. 1239 Siping Road, Yangpu District, Shanghai 200092, PR China. E-mail: duai@ tongji.edu.cn; zhoubin863@tongji.edu.cn pressure drying of PMSQ gel does not need the surface modification since PMSQ gel system contains abundent methyl groups. Hayase et al. studied the spring-back behavior during evaporative drying ${ }^{18}$ and found that it was difficult to obtain aerogels with densities of $c a .140 \mathrm{mg} \mathrm{cm}^{-3}$ due to irreversible shrinkage. ${ }^{18}$ Thus, they used an extended ageing process to improve the mechanical strength of the PMSQ gel and let it spring back to its original size. ${ }^{\mathbf{1 8}}$ They also found that the silanol groups in the PMSQ network were reduced by this ageing process. ${ }^{18}$ Thus, we hypothesize that the reduction in irreversible shrinkage could be due to the decrease of surface silanol groups, rather than the effect of skeleton strengthening. The surface modification process for silica aerogels had been well studied. ${ }^{20-23}$ In this paper, we applied a mature surface modification technique to add terminal silane groups to PMSQ gels to reduce surface silanol groups without strengthening the skeletons. We used trimethylsilyl groups with three non-hydrolysable $-\mathrm{CH}_{3}$ terminal groups to react with the surface silanol groups of the PMSQ gel. Unlike the ageing process, surface modification with terminal silane groups will not increase the density. Thus, this technique should be helpful for preparing low-density PMSQ aerogels under ambient pressure drying.

The results show that the surface modification process can reduce irreversible shrinkage and allow the PMSQ gels to spring back to their original sizes. Without using the extended ageing 


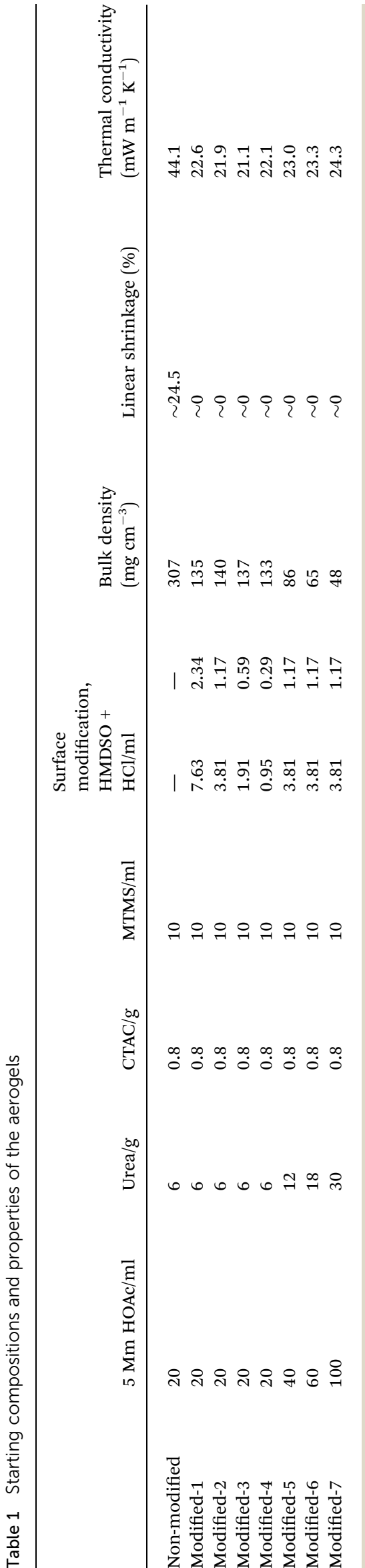

process, aerogels with very low densities were obtained. The aerogel with a density of $c a .48 \mathrm{mg} \mathrm{cm}^{-3}$ is currently the lowest density PMSQ aerogel obtained by ambient pressure drying; this density is similar to the lowest reported density of $\sim 35 \mathrm{mg} \mathrm{cm}^{-3}$ obtained via supercritical fluid drying. ${ }^{24}$ Aerogels with very low density, high transparency, low thermal conductivity and superhydrophobicity could be obtained by this method without extend ageing. We also found that the PMSQ gels still contained a considerable amount of surface hydroxyl groups. The main driving force of irreversible shrinkage was believed to be continuous condensation between surface hydroxyl groups due to capillary force during drying. ${ }^{25}$ The method proposed herein greatly improves the efficiency of ambient pressure dying for crack-free PMSQ aerogels.

\section{Experimental}

\subsection{Preparation of PMSQ gels}

The PMSQ gels were prepared by a two-step, acid/base-catalyzed sol-gel process reported by Kanamori et al. ${ }^{13}$ First, $x \mathrm{ml}$ of $5 \mathrm{mM}$ aqueous acetic acid, $0.3 \times x \mathrm{~g}$ of urea (Sinopharm Chemical Reagent Co., Ltd), and $0.8 \mathrm{~g}$ of surfactant CTAC (Sinopharm Chemical Reagent Co., Ltd) were dissolved in a glass beaker, and $10.0 \mathrm{ml}$ of MTMS (Sinopharm Chemical Reagent Co., Ltd) was then added under vigorous stirring. Subsequently, the solution was stirred for $30 \mathrm{~min}$ at room temperature for acidcatalyzed hydrolysis followed by base-catalyzed gelation at $60{ }^{\circ} \mathrm{C}$ and ageing for $2 \mathrm{~d}$.

\subsection{Solvent exchange/surface modification and ambient drying process}

After ageing, the PMSQ gels were first washed in methanol (Sinopharm Chemical Reagent Co., Ltd). The methanol was exchanged with fresh methanol three times every 12 hours. The gels were then washed in 2-propanol (Sinopharm Chemical Reagent Co., Ltd) three times every 12 hours to remove CTAC. Subsequently, the PMSQ gels were chemically modified by 2 propanol (Sinopharm Chemical Reagent Co., Ltd)/HMDSO (Hangzhou Guibao Chemical Co., Ltd, China)/HCL (36.4\%, Sinopharm Chemical Reagent Co., Ltd) solutions. In this study, $200 \mathrm{ml}$ 2-propanol was used as the solvent. Different amounts of HMDSO were added to the solution. The HMDSO : HCL molar ratio was kept constant at $5: 4$.

After the surface modification process, the PMSQ gels were washed in HMDSO solution exchanged with fresh solution three times every 12 hours. During the drying process, HMDSO was slowly removed by evaporation at room temperature for $1 \mathrm{~d}$. After drying, the aerogels were heated at $90^{\circ} \mathrm{C}$ for $2 \mathrm{~h}$ and $160^{\circ} \mathrm{C}$ for another $2 \mathrm{~h}$ to allow the further spring-back of the gel network. Modified-7 could not spring back further after heating at $160{ }^{\circ} \mathrm{C}$. Thus, Modified- $7\left(48 \mathrm{mg} \mathrm{cm}^{-3}\right)$ was further heated at $300{ }^{\circ} \mathrm{C}$ for $1 \mathrm{~h}$ to allow further spring-back of the gel network.

\subsection{Characterization}

The bulk density was obtained by measuring the volume and weight of the aerogel. The pore structure was investigated by the 
standard $\mathrm{N}_{2}$ gas adsorption method using a surface area analyzer (AUTOSORB-1-MP, Quantachrome). Initially, the PMSQ aerogels were degassed at $120{ }^{\circ} \mathrm{C}$ for $10 \mathrm{~h}$, and the adsorption-desorption isotherms were obtained at $77 \mathrm{~K}$. The surface areas were calculated using the Brunauer-Emmett-Teller (BET) method. The average pore size and total pore volume were calculated from the BET analysis. The pore size distributions were estimated by the BarrettJoyner-Halenda (BJH) method. The degrees of hydrophobicity were quantified by measuring the contact angle of a water droplet placed on a monolith surface. Fourier transform infrared spectroscopy (FT-IR, TENSOR 27, Germany) was used for chemical characterization. The directional transmittances were measured by UV-Vis-IR spectrophotometry (JASCO V-570). The microstructures of the PMSQ aerogels were observed by scanning electron microscopy (SEM; XL30FEG, Netherlands). Compression tests were conducted on a CMT 5105 universal materials testing machine under room conditions with a constant compression speed at $10 \%$ of the sample length per minute. ${ }^{26}$ To assess the molecular-level structure of the obtained PMSQ aerogel, ${ }^{29} \mathrm{Si}$ MAS NMR experiments were performed on an Agilent 600 DD2 spectrometer at a resonance frequency of $119.13 \mathrm{MHz} .{ }^{29} \mathrm{Si} \mathrm{NMR}$ spectra were recorded with spinning rate of $7 \mathrm{kHz}$ with a $6 \mathrm{~mm}$ probe at room temperature. ${ }^{29} \mathrm{Si}$ DDMAS experiments were performed with a delay time of $5 \mathrm{~s}$. Chemical analysis on the surface elements were recorded on an X-ray photoelectron spectrometer (USA ThermoFischer, ESCALAB 250Xi). The background pressure during analysis was $8 \times 10^{-10} \mathrm{~Pa}$. An aluminum $\mathrm{K} \alpha$ ray $(h \nu=$ $1486.6 \mathrm{eV}$ ) was used as the X-ray radiation source. Curve deconvolution of the obtained XP spectra was performed with an XPS peak-fitting program (XPSPEAK41, Chemistry, CUHK).

\section{Results and discussion}

\subsection{Detailed parameters of PMSQ aerogels}

The starting compositions and properties of all the aerogels are shown in Table 1. As shown in Table 1, the surface modification process clearly reduced the irreversible shrinkage. All the modified samples sprung back to their original sizes. Compared to the modified aerogels, the non-modified gels exhibited a linear shrinkage of $24.5 \%$, resulting in higher density $\left(\sim 307 \mathrm{mg} \mathrm{cm}^{-3}\right)$. In addition, the gel treated with a small amount of $\mathrm{HMDSO} / \mathrm{HCl}$ (Modified-4) still had the ability to spring back to its original size. A low-density PMSQ aerogel (48 $\mathrm{mg} \mathrm{cm}^{-3}$ ) was obtained by ambient pressure drying; this density is similar to the lowest reported density obtained via supercritical fluid drying. ${ }^{18,24}$ Fig. 1a shows 14 PMSQ aerogels prepared by this method. The difficulties in obtaining a good monolith and low density have been solved to a large extent. Fig. 1b shows a photograph of Modified-7 (left) and Modified-4 (right). Fig. 1b shows that Modified-4 shows fine transparency and much more transparency than Modified-7.

\subsection{Mechanism of surface modification}

To reduce irreversible shrinkage and obtain a highly porous structure, the $\mathrm{Si}-\mathrm{OH}$ groups should be modified with -O-Si$\left(\mathrm{CH}_{3}\right)_{3}$. In this study, hydrochloric acid promoted the hydrolysis

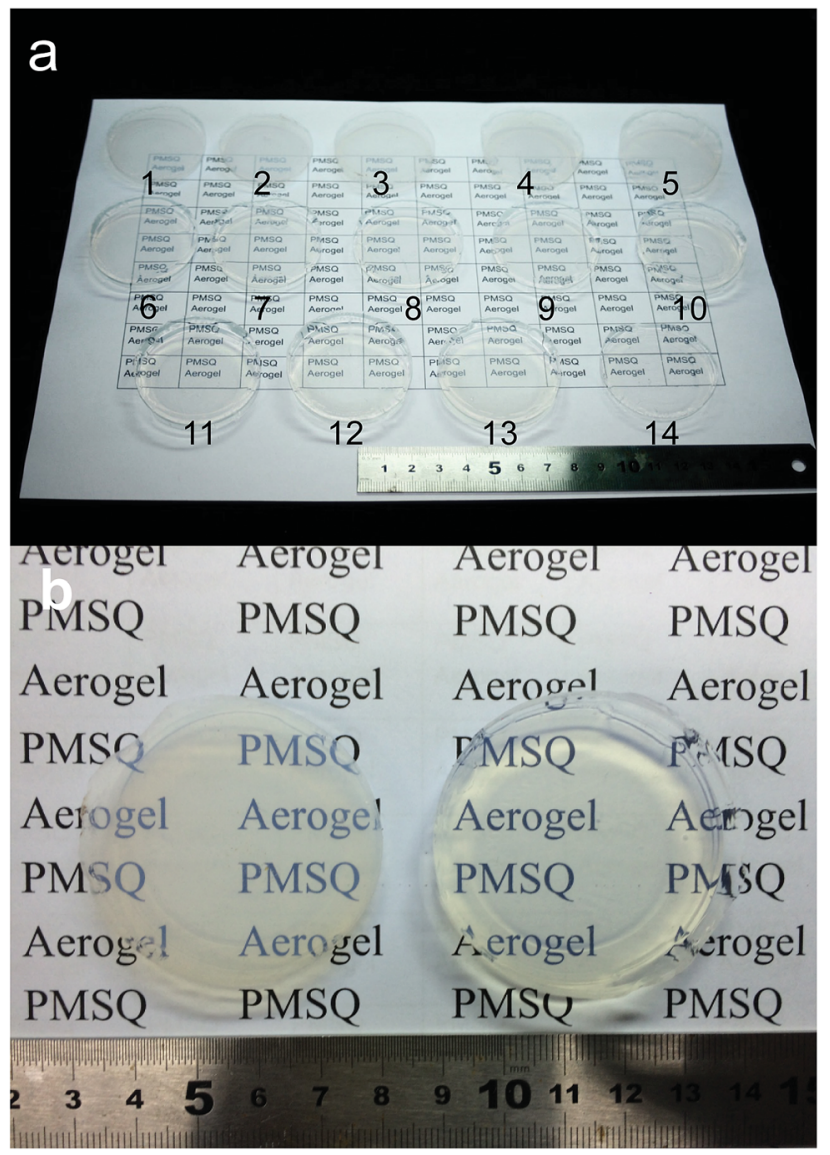

Fig. 1 (a) Fourteen PMSQ aerogels prepared by the proposed method: (1-3) aerogels with densities of $\sim 48 \mathrm{mg} \mathrm{cm}^{-3}$; (4-5) aerogels with densities of $\sim 64 \mathrm{mg} \mathrm{cm}^{-3}$; (6-10) aerogels with densities of $\sim 84 \mathrm{mg}$ $\mathrm{cm}^{-3}$; and (11-14) aerogels with densities of $\sim 140 \mathrm{mg} \mathrm{cm}^{-3}$. (b) Photograph of Modified-7 (left) with a density of $\sim 48 \mathrm{mg} \mathrm{cm}^{-3}$ and Modified-4 (right) with a density of $133 \mathrm{mg} \mathrm{cm}^{-3}$.

of HMDSO to form trimethylchlorosilane (TMCS; eqn (1)). HMDSO/HCL (TMCS) also reacts with pore water and $\mathrm{Si}-\mathrm{OH}$ groups (eqn (2)) on the surfaces of alcogels. ${ }^{23,27}$ 2-Propanol was used as the solvent to reduce the capillary stress and help slow the reaction rate of TMCS with pore water. ${ }^{23}$

$$
\begin{aligned}
& \left(\mathrm{CH}_{3}\right)_{3}-\mathrm{Si}-\mathrm{O}-\mathrm{Si}-\left(\mathrm{CH}_{3}\right)_{3}[\mathrm{HMDSO}]+2 \mathrm{HCl} \rightarrow 2\left(\mathrm{CH}_{3}\right)_{3} \mathrm{Si}-\mathrm{Cl} \\
& {[\mathrm{TMCS}]+\mathrm{H}_{2} \mathrm{O}} \\
& \quad \equiv \mathrm{Si}-\mathrm{OH}+\left(\mathrm{CH}_{3}\right)_{3} \mathrm{Si}-\mathrm{Cl} \rightarrow \equiv \mathrm{Si}-\mathrm{O}-\mathrm{Si}-\left(\mathrm{CH}_{3}\right)_{3}+\mathrm{HCl}
\end{aligned}
$$

The effect of surface modification was observed using FT-IR. Fig. 2 shows the FT-IR spectra of all the PMSQ aerogels in air at room temperature in the region of $400-4000 \mathrm{~cm}^{-1}$. Compared to the non-modified aerogel, the absorption bands of all modified samples at $920 \mathrm{~cm}^{-1}$ in the FT-IR spectra were decreased. This indicates that the unreacted silanol groups in the PMSQ network were effectively reduced by surface modification. ${ }^{\mathbf{1 4 , 1 8}}$ The peak at $1638 \mathrm{~cm}^{-1}$ and the broad absorption band attributed to $-\mathrm{OH}$ groups at around $3500 \mathrm{~cm}^{-1}$ are due to adsorbed water. ${ }^{28-31}$. The PMSQ aerogels have peaks at around $2975 \mathrm{~cm}^{-1}$ 


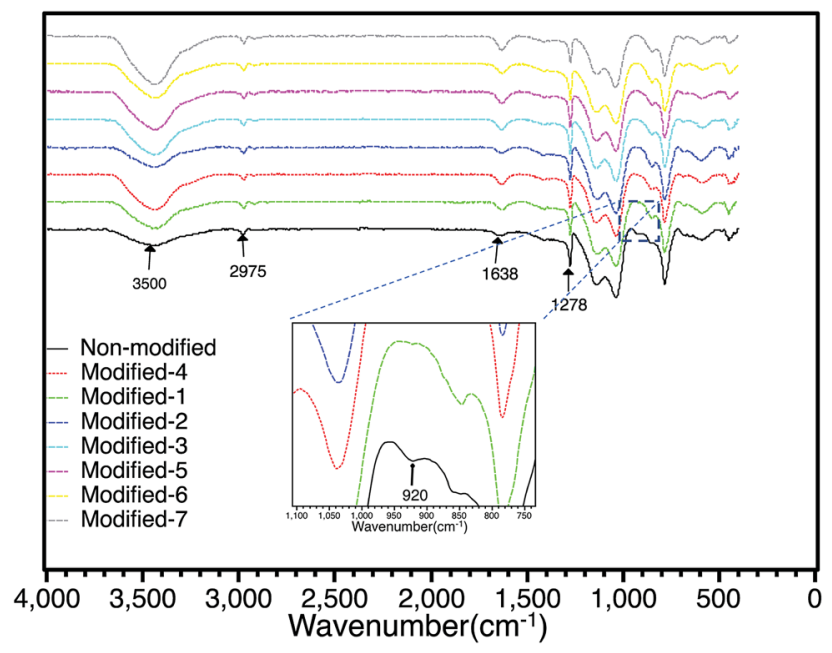

Fig. 2 FT-IR spectra of PMSQ aerogels.

and $1278 \mathrm{~cm}^{-1}$ corresponding to $\mathrm{Si}-\mathrm{CH}_{3}$ asymmetric bending. ${ }^{32}$ In the non-modified aerogel, the $\mathrm{Si}-\mathrm{CH}_{3}$ groups are derived from the non-hydrolyzable methyl groups $\left(-\mathrm{CH}_{3}\right)$ in the MTMS precursor. For the Modified- 4 aerogel, the $\mathrm{Si}-\mathrm{CH}_{3}$ groups are derived from the MTMS precursor $\left(\mathrm{Si}-\mathrm{CH}_{3}\right)$ and HDMSO $\left(\mathrm{Si}-\left(\mathrm{CH}_{3}\right)_{3}\right)$.

${ }^{29} \mathrm{Si}$ MAS NMR was adopted in this study to obtain the molecular-level structure of the modified and non-modified PMSQ aerogels. The ${ }^{29} \mathrm{Si}$ NMR spectra of the Modified-4 and non-modified samples are shown in Fig. 3. The ${ }^{29} \mathrm{Si}$ NMR spectrum of non-modified had ${ }^{29}$ SiNMR signals at about -48 , -56 , and $-67 \mathrm{ppm}$, corresponding to $\left.\mathrm{T}^{1}(\mathrm{HO})_{2} \mathrm{Si}(\mathrm{OSi}) \mathrm{CH}_{3}\right), \mathrm{T}^{2}$ ( $\left.\mathrm{HO}) \mathrm{Si}(\mathrm{OSi})_{2} \mathrm{CH}_{3}\right)$, and $\mathrm{T}^{3}\left(\mathrm{Si}(\mathrm{OSi})_{2} \mathrm{CH}_{3}\right){ }^{33,34}$ Based on the ${ }^{29} \mathrm{Si}$ MAS NMR spectrum, we can conclude that considerable hydroxyl groups still existed on the surface of the network of the non-modified aerogel (from $\mathrm{T}^{1}$ and $\left.\mathrm{T}^{2}\right){ }^{34}$ The relative intensities of the $\left.\mathrm{T}^{1}(\mathrm{HO})_{2} \mathrm{Si}(\mathrm{OSi}) \mathrm{CH}_{3}\right)$ and $\mathrm{T}^{2}(\mathrm{HO}) \mathrm{Si}(\mathrm{OSi})_{2} \mathrm{CH}_{3}$ ) peaks were decreased in the NMR spectrum of Modified-4, which indicates that the hydroxyl groups on the surface of PMSQ gel network were effectively reacted with modification agents $\left(-\mathrm{Si}\left(\mathrm{CH}_{3}\right)_{3}\right)$.

The XPS spectra of non-modified and Modified-4 are presented in Fig. 4 for the analysis of chemical elements on the

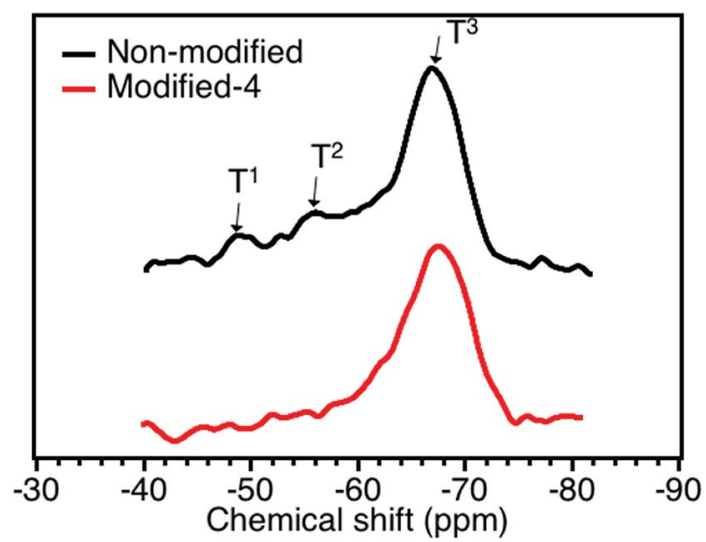

Fig. $3{ }^{29}$ Si NMR spectra of Modified-4 and non-modified. sample surfaces. Three photoelectron peaks located at 284.5, 532.4, and $102.6 \mathrm{eV}$, corresponding to $\mathrm{C} 1 \mathrm{~s}$, O1s and Si2p, respectively, were detected in the XPS spectra of non-modified and Modified-4..$^{34,35}$ The 01s spectra of non-modified and Modified-4 can be divided into two peaks, 532.4 and $533.1 \mathrm{eV}$, which correspond to $\mathrm{SiO}_{2}^{*}$ and $\mathrm{O}^{*} \mathrm{H}$ units, respectively. ${ }^{34,36}$ The ratios of $\mathrm{O}^{*} \mathrm{H} / \mathrm{SiO}_{2}^{*}$ were calculated from the peak area ratios to be $40.3 \%$ and $15.2 \%$ for non-modified and Modified-4, respectively. The smaller $\mathrm{O}^{*} \mathrm{H} / \mathrm{SiO}_{2}^{*}$ ratio of Modified-4 is attributed to the reaction of hydroxyl groups with the modification agents. The Si $2 \mathrm{p}$ spectrum is also divided into two peaks, one at $102.4 \mathrm{eV}$ and another at $103 \mathrm{eV}$. The former is attributed to $\mathrm{O}-$ $\mathrm{Si}^{*}-\mathrm{CH}_{3}$ groups, while the latter is the characteristic peak of the $\mathrm{Si}$ of $\mathrm{Si}^{*} \mathrm{O}_{2} .{ }^{34}$ The $\mathrm{O}-\mathrm{Si}^{*}-\mathrm{CH}_{3} / \mathrm{Si}^{*} \mathrm{O}_{2}$ ratios of non-modified and Modified- 4 were $35.2 \%$ and $43.8 \%$, respectively. The larger O$\mathrm{Si}^{*}-\mathrm{CH}_{3} / \mathrm{Si}^{*} \mathrm{O}_{2}$ ratio is attributed to the additional $\mathrm{O}-$ $\mathrm{Si}^{*}-\left(\mathrm{CH}_{3}\right)_{3}$ groups from the modification agents.

The $\mathrm{Si}-\mathrm{CH}_{3}$ groups result in a hydrophobic aerogel. The hydrophobicity was characterized by measuring the contact angle of water. Fig. 5 shows the water droplets placed on the surfaces of modified and non-modified aerogels. The contact angles of Modified-4 and non-modified were $153^{\circ}$ and $93^{\circ}$, respectively. The low contact angle of non-modified may be due to its high density $\left(\sim 0.3 \mathrm{~g} \mathrm{~cm}^{-3}\right)$. Super-hydrophobicity is normally derived from high nanoscale roughness and groups with low surface energy. The surfaces of aerogels with high density are flat and similar to the surfaces of plastics. Fig. 6a shows the relatively dense structure of the non-modified aerogel, which may explain the low contact angle. Modified-4 also shows a high contact angle $\left(153^{\circ}\right)$ compared to previously reported samples $\left(140^{\circ}\right)$ with the same starting compositions. ${ }^{17}$ The improved contact angle in this study is attributed to the surface modification, which clearly improved the hydrophobicity of the PMSQ aerogel. Modified-1, Modified-2, Modified-3, and Modified-4, which were modified with different amounts of modification agent (Table 1), showed similar contact angles. Thus, modification agents with $0.95 \mathrm{ml} \mathrm{HMDSO}$ and $0.29 \mathrm{ml} \mathrm{HCl}$ can effectively react with the hydroxyl groups in the gel with a target density of $\sim 140 \mathrm{mg} \mathrm{cm}^{-3}$. However, to react the hydroxyl groups on the surfaces of the low-density samples, we used $3.81 \mathrm{ml}$ HMDSO and $1.17 \mathrm{ml} \mathrm{HCl}$.

\subsection{Microstructure}

The microstructures of the silica aerogels were investigated by SEM. The pore structures were investigated by $\mathrm{N}_{2}$ gas adsorption.

Fig. 6 shows the SEM micrographs of the PMSQ aerogels. Non-modified, Modified-4 and Modified-5 exhibited typical string-of-pearls morphologies and globular aggregated structures. ${ }^{37}$ The clusters of non-modified were densely packed together, indicating structural collapse during ambient pressure drying. ${ }^{38}$ Modified-4 had larger pores than non-modified as a result of the surface modification; Modified-4 was able to spring back to its original size, resulting in a porous structure with lower density. Modified-6 and Modified-7 showed continuous branched networks with many macropores, typical for the 

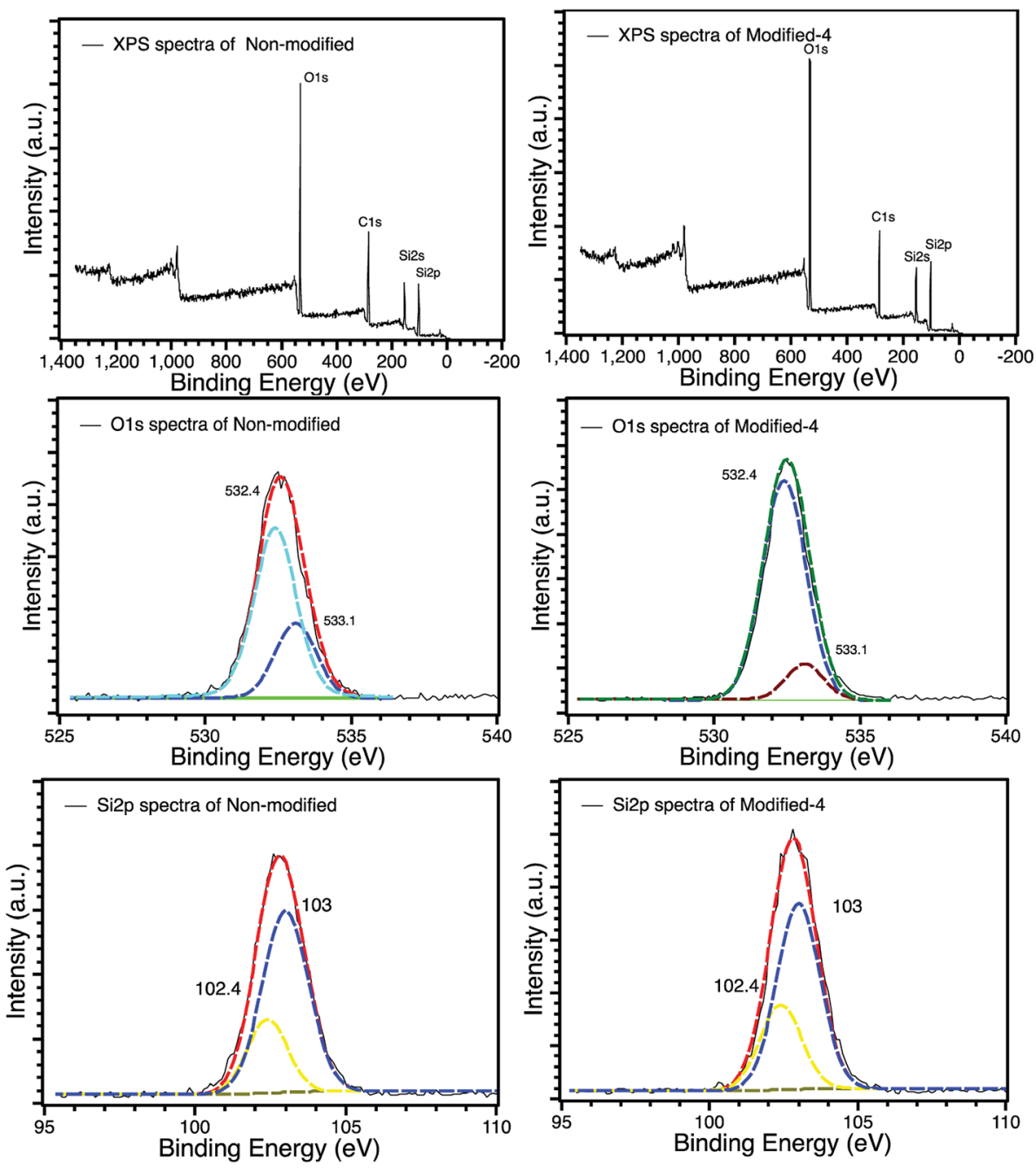

Fig. 4 XPS spectra of non-modified and Modified-4.

networks of low-density PMSQ aerogels. ${ }^{24}$ These slender structures make it difficult for the aerogels to spring back to their original sizes at $200{ }^{\circ} \mathrm{C}$. Thus, thermal treatment at higher temperature is critical for obtaining low-density aerogels. Increasing the temperature to $300{ }^{\circ} \mathrm{C}$ can increase the repulsion between methyl groups, allowing Modified- 6 and Modified-7 to completely spring back to their original sizes. This method is useful for obtaining PMSQ aerogels with low density.

Fig. 7a shows the nitrogen adsorption/desorption isotherms of aerogels. All aerogels exhibited lower adsorption at low pressure, and adsorption monotonically increased with increasing relative pressure, which is characteristic of type IV isotherms. $^{37,39}$ Modified-1, Modified-2, Modified-3 and Modified-4 showed similar nitrogen adsorption/desorption isotherms. The hysteresis loops of the modified aerogels were larger than that of the non-modified aerogel. These larger hysteresis loops indicate the existence of more pores in the silica network. ${ }^{23}$ The adsorption isotherms of Modified- 6 and Modified-7 showed rapid increases in the region of high relative pressure ( $>0.95$ ), indicating that Modified- 6 and Modified- 7 had very porous structures. Fig. $7 \mathrm{~b}$ shows the pore size distribution. The surface area, total pore volume and average pore size are shown in Table 2. As shown in Fig. 7b, the non-modified aerogel showed a peak at $\sim 17 \mathrm{~nm}$. Compared to the non-modified aerogel, Modified-1, Modified-2, Modified-3 and Modified-4 had larger peaks at $\sim 23 \mathrm{~nm}$. Modified-5 had a peak at $33 \mathrm{~nm}$. Modified- 6 and Modified-7 had no peaks in the region from 0$100 \mathrm{~nm}$ due to the limits of the $\mathrm{N}_{2}$ gas adsorption/desorption method. Pores larger than $100 \mathrm{~nm}$ could not be detected. Table 2 also shows that the PMSQ aerogels with lower densities had larger average pore sizes. Fig. 6, 7b, and Table 2 all demonstrate that very porous structures were obtained by ambient pressure drying cum surface modification process.

On the other hand, the thermal conductivity of PMSQ aerogels were affected by the porous structures and the densities. The thermal conductivity of a porous material consists of three components: heat transfer by the gas phase, solid phase and radiation. ${ }^{18}$ The thermal conductivities (Table 1) of Modified-1, Modified-2, Modified-3 and Modified-4 (ca. $22 \mathrm{~mW} \mathrm{~m}^{-1} \mathrm{~K}^{-1}$ ) are much lower than non-modified $\left(44.1 \mathrm{~mW} \mathrm{~m}^{-1} \mathrm{~K}^{-1}\right)$. The reason for this is that density of these aerogels $\left(\sim 140 \mathrm{mg} \mathrm{cm}^{-3}\right)$ are much lower than non-modified $\left(\sim 307 \mathrm{mg} \mathrm{cm}^{-3}\right)$. In this case, heat transfer by solid phase is the major part that affects the thermal conductivity. ${ }^{18}$ The samples Modified- 6 and Modified-7 


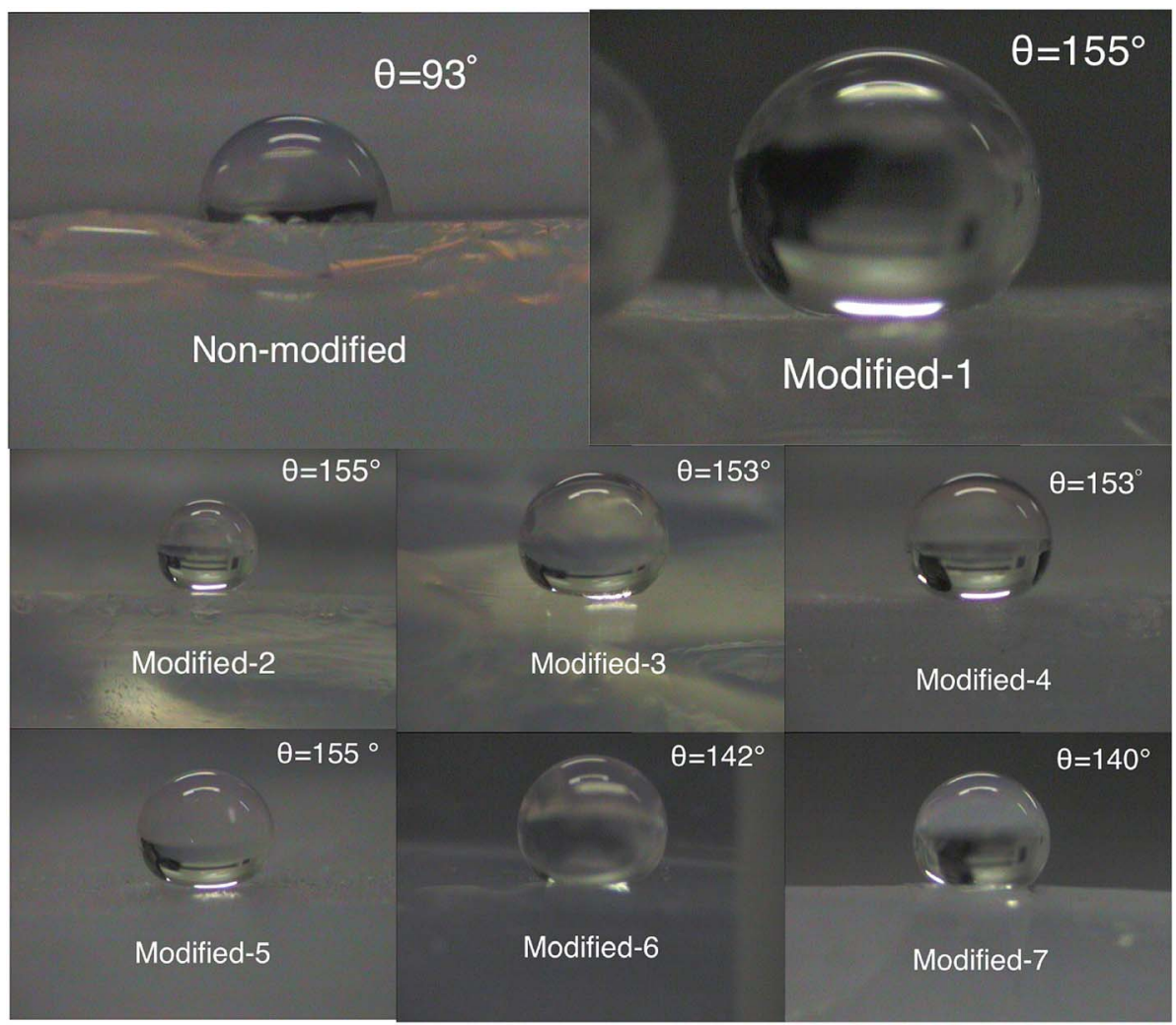

Fig. 5 Water contact angles of PMSQ aerogels.

show relatively lower density but higher thermal conductivity since they have more macropores in the network (Fig. 6). Although they have lower density. The increasing amount of macropores in the network will lead to the increase of heat transfer by the gas phase.

\subsection{Light transmittance}

Directional transmittance was measured using an ultravioletvisible-infrared (UV-Vis-IR) spectrophotometer (JASCO V-570). The thicknesses of all modified samples were approximately
$10 \mathrm{~mm}$, while that of non-modified was approximately $7 \mathrm{~mm}$. Fig. 8 shows the directional transmittances of all samples. The transmittance of Modified-4 at $550 \mathrm{~nm}$ was $81.3 \%$, while that of non-modified was $42.9 \%$. The transmittance of Modified- 4 at $550 \mathrm{~nm}$ was also higher than those of Modified-5 (72.7\%), Modified-6 (57.4) and Modified-7 (50.4\%). At the short wavelength $(400 \mathrm{~nm})$, Modified-4 had a transmittance of $52.8 \%$, much higher than those of non-modified (8.5\%), Modified-5 (36.3\%), Modified-6 (22.6\%) and Modified-7 (14.9\%). The optical transmittance of a silica aerogel is directly affected by light scattering. ${ }^{40}$ This includes scattering caused by
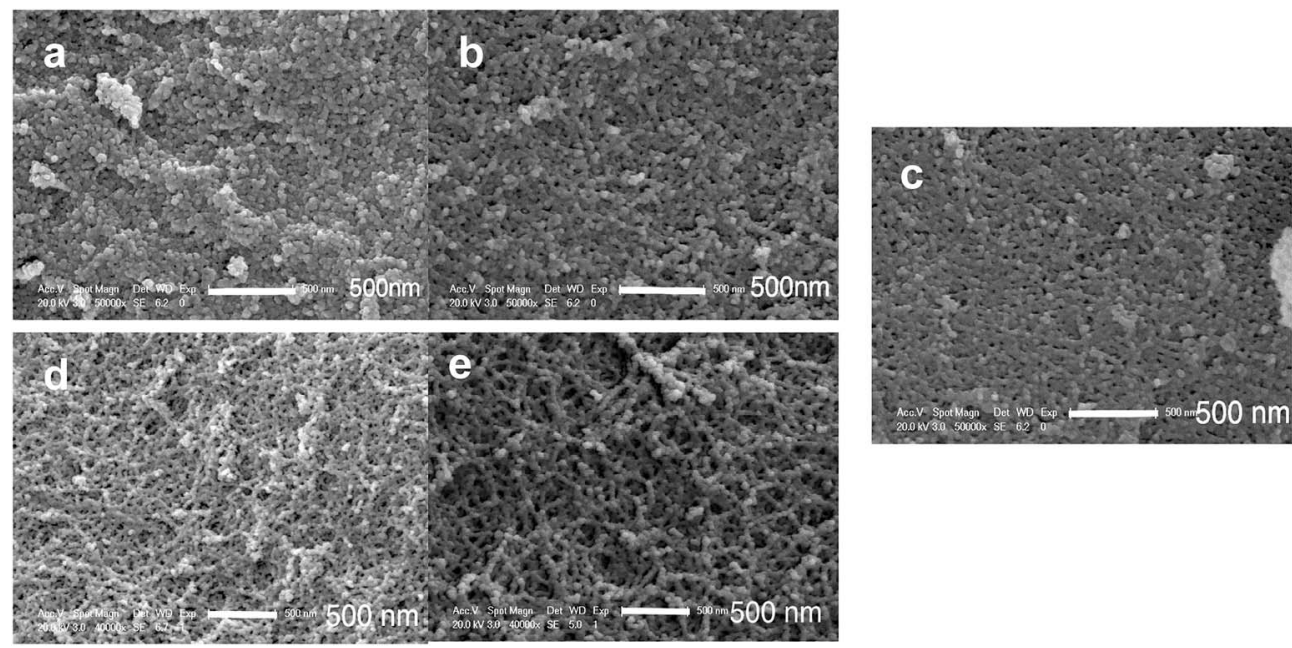

Fig. 6 SEM micrographs of PMSQ aerogels: (a) non-modified, (b) Modified-4, (c) Modified-5, (d) Modified-6 and (e) Modified-7. 

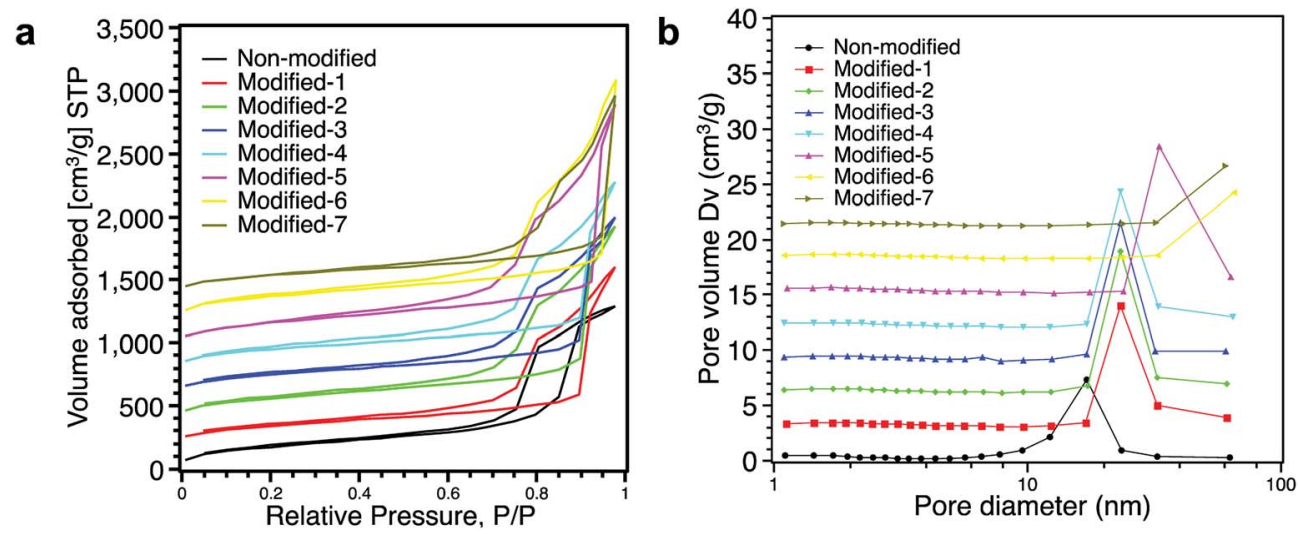

Fig. 7 (a) Nitrogen adsorption/desorption isotherms and (b) pore size distributions of all the aerogels.

micrometer-size imperfections in the external aerogel surface, which explains the slightly fuzzy appearance of objects viewed through an aerogel, and scattering from the nanoporous aerogel network. The Rayleigh scattering mainly occurs since the pore and cluster sizes of aerogels are much smaller than the wavelengths of the light. ${ }^{40}$ The main reason for the higher transmittance of Modified-4 compared to non-modified is the bulk density; ${ }^{13}$ non-modified had a higher density than Modified-4. A higher bulk density leads to more scattering sources inside the gel body. ${ }^{13,41}$ It also should be mentioned that the transmittances of Modified-6 and Modified-7 were lower than that of Modified-4 because the larger pore sizes of Modified-6 and Modified-7 led to increased light scattering. Compared to Modified-1, -2 and -3, Modified-4 showed a lower transmittance in the UV region, possibly because of the larger clusters of Modified-4. The reason for its higher transmittance at wavelengths greater than $500 \mathrm{~nm}$ may indicate a slightly lower surface reflectivity for Modified-4. However, the differences between the optical transmissions of Modified-4 and Modified-1, -2 and -3 were small. Modified-1, $-2,-3$ and -4 had high transmittances in the visible region $(380-780 \mathrm{~nm})$. These high-transparency aerogels with low thermal conductivities are suitable for transparent insulating materials.

\subsection{Mechanical properties}

Due to their extreme brittleness, silica aerogels have not been widely used. The drawback of silica aerogels is their extreme

Table 2 Surface area, total pore volume and average pore size of all aerogels

\begin{tabular}{llll}
\hline & $\begin{array}{l}\text { Surface area } \\
\left(\mathrm{m}^{2} \mathrm{~g}^{-1}\right)\end{array}$ & $\begin{array}{l}\text { Total pore volume } \\
\left(\mathrm{cm}^{3} \mathrm{~g}^{-1}\right)\end{array}$ & $\begin{array}{l}\text { Average pore } \\
\text { size }(\mathrm{nm})\end{array}$ \\
\hline Non-modified & 637 & 2.1 & 12.6 \\
Modified-1 & 526 & 2.2 & 15.8 \\
Modified-2 & 597 & 2.4 & 15.8 \\
Modified-3 & 552 & 2.2 & 15.7 \\
Modified-4 & 552 & 2.3 & 16.6 \\
Modified-5 & 619 & 2.9 & 18.9 \\
Modified-6 & 627 & 2.9 & 18.9 \\
Modified-7 & 506 & 2.4 & 19.1
\end{tabular}

brittleness, which arises from the high porosity and low connectivity of each colloidal particle. ${ }^{14}$ PMSQ aerogels derived from MTMS show drastic improvements in mechanical durability against compression. ${ }^{14}$ Fig. 9a shows the spring-back phenomenon during the uniaxial compression test of Modified-4. The gel shrank up to $70 \%$ on a linear scale and sprung back to its original size when unloaded. Fig. 9b displays the spring-back phenomenon of Modified-5 during drying. The gel shrank up to $42 \%$ on a linear scale and then sprang back to its original size after heating at $160{ }^{\circ} \mathrm{C}$. Fig. 9c shows the stressstrain curves of non-modified and Modified-4. The Young's modulus of each sample was evaluated as the initial linear slope of the stress-strain curve. ${ }^{26,42}$ The Young's moduli of nonmodified, Modified-4, Modified-5, Modified-6 and Modified-7 were $1.5,1.07,0.49,0.31$ and $0.12 \mathrm{MPa}$, respectively. In this case, the modulus was mainly affect by the density. PMSQ aerogel with a lower density has a lower modulus. As shown in Fig. 9c, the stress of non-modified aerogel showed a linear increase at the strain range of $0-4 \%$. Non-modified collapsed at a strain of approximately $27 \%$, but could be continuously measure until reaching a strain of $\sim 41 \%$. Modified- 4 showed a linear increase from $0-20 \%$. Modified- 7 had a linear increase from $0-50 \%$. This indicates that Modified-7 had a softer and

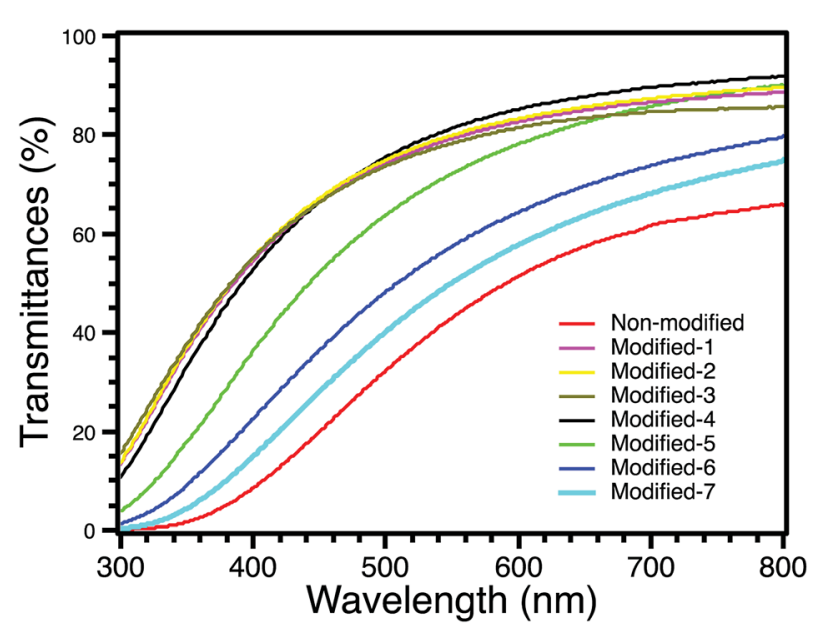

Fig. 8 Directional transmittances of all the aerogels. 
a

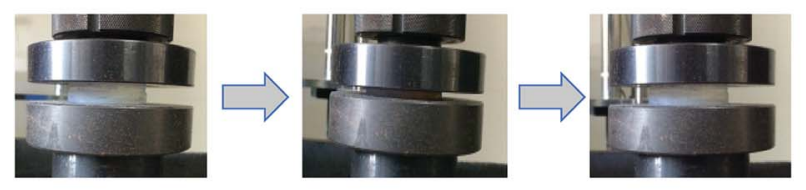

b
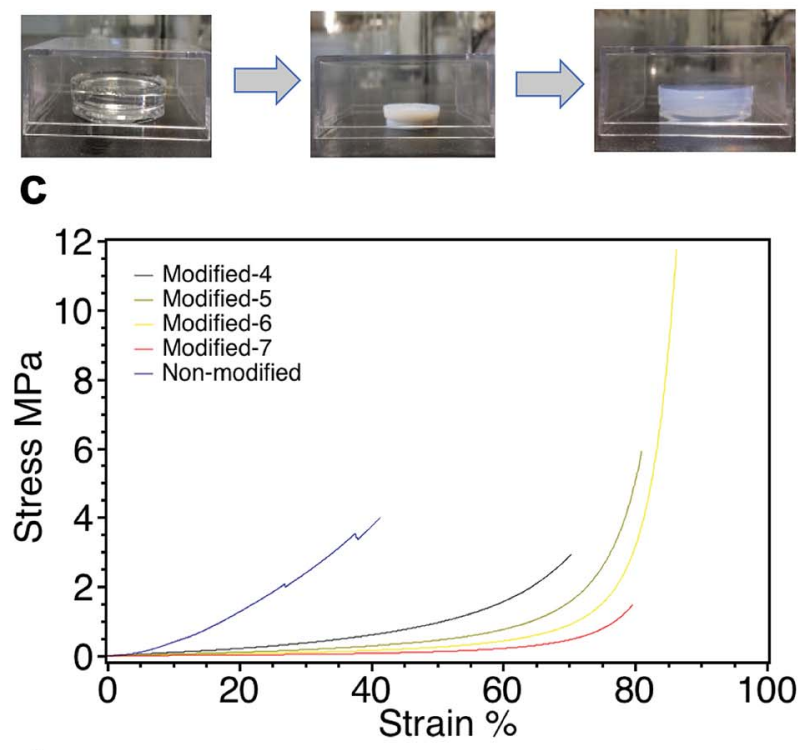

d
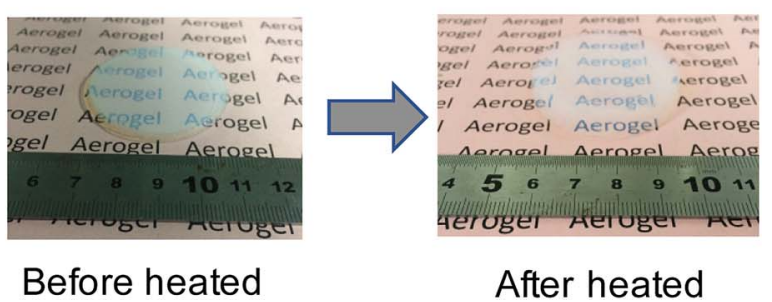

After heated

Fig. 9 Images showing (a) the spring-back phenomenon during a uniaxial compression test of Modified-4 and (b) the spring-back phenomenon of Modified-5 during ambient pressure drying. (c) Compressive stress-strain curves of PMSQ aerogels. (d) Images showing the spring-back phenomenon of compressed Modified-7 after heating at $300^{\circ} \mathrm{C}$.

more continuous skeleton that could undergo substantial deformation.

Modified-4 exhibited a higher deformability (up to $70 \%$ strain) than non-modified (up to $41 \%$ ) and spontaneously recovered to its original size without breaking when the pressure was released. The methyl groups from the precursor MTMS and the surface modifications were homogeneously distributed with high density in the network, which induces the repulsion interaction among the shrunk skeletons of the gel. ${ }^{14}$ In contrast, non-modified underwent irreversible shrinkage during ambient pressure drying, leading to its high density and stiffness. Modified-5, Modified-6 and Modified-7 exhibited higher deformability (up to $80 \%$ strain) than Modified-4. However, Modified-5, Modified-6 and Modified-7 only sprang back to
$73 \%, 43 \%$ and $29 \%$ of their original sizes, respectively. Surprisingly, after compression, these samples nearly sprang back to their original sizes after heating at $300{ }^{\circ} \mathrm{C}$ for $1 \mathrm{~h}$. Fig. $9 \mathrm{~d}$ shows Modified-7 recovered to its initial size after heating at $300{ }^{\circ} \mathrm{C}$.

\section{Conclusions}

We applied a mature technique to modify the surfaces of PMSQ aerogels with terminal silane groups containing three nonhydrolysable $-\mathrm{CH}_{3}$ groups. The results show that the surface modification process can reduce irreversible shrinkage and allow the gels spring back to their original sizes. The gels treated with a small amount of surface modification solution still had the ability to spring back to their original sizes. Considerable $\mathrm{Si}-\mathrm{OH}$ groups still remained in the PMSQ gels. The irreversible shrinkage was primarily attributed to the condensation between surface $\mathrm{Si}-\mathrm{OH}$ groups. The surface modification process greatly reduced the amount of Si-OH groups in the PMSQ gel system. The problem of obtaining a good monolith with low density and without irreversible shrinkage or cracking has been solved to a large extent. Aerogels with a low density of $48 \mathrm{mg} \mathrm{cm}^{-3}$, low thermal conductivity $\left(21.1 \mathrm{~mW} \mathrm{~m}^{-1} \mathrm{~K}^{-1}\right)$, high transparency $(81.3 \%$ at $550 \mathrm{~nm})$, super-hydrophobicity (contact angle of $155^{\circ}$ ) and excellent mechanical properties will be useful for the industrial production of transparent insulating materials.

\section{Conflicts of interest}

The authors declare that they have no competing interests.

\section{Acknowledgements}

This research is financially supported by the National Key Research and Development Program of China (2017YFA0204600), Science and Technology Innovation Fund of Shanghai Aerospace, China (SAST201469) and Sheng Feiyun Science and Technology Innovation Fund of Tongji University.

\section{References}

1 N. Hüsing and U. Schubert, Angew. Chem., Int. Ed., 1998, 37, 22-45.

2 H. Ren, X. Shi, J. Zhu, Y. Zhang, Y. Bi and L. Zhang, J. Mater. Sci., 2016, 51, 6419-6427.

3 I. Saptiama, Y. V. Kaneti, H. Oveisi, Y. Suzuki, K. Tsuchiya, K. Takai, T. Sakae, S. Pradhan, M. S. A. Hossain, N. Fukumitsu, K. Ariga and Y. Yamauchi, Bull. Chem. Soc. Jpn., 2018, 91, 195-200.

4 J. Zhu, X. Yang, Z. Fu, J. He, C. Wang, W. Wu and L. Zhang, Chem.-Eur. J., 2016, 22, 2515-2524.

5 S. Li, H. Ren, J. Zhu, Y. Bi, Y. Xu and L. Zhang, J. Non-Cryst. Solids, 2017, 473, 59-63.

$6 \mathrm{~J}$. Fricke and A. Emmerling, J. Am. Ceram. Soc., 1992, 75, 2027-2035.

7 J. Fricke, J. Non-Cryst. Solids, 1988, 100, 169-173.

8 C. E. Carraher, Polym. News, 2005, 30, 386-388. 
9 J. M. Schultz, K. I. Jensen and F. H. Kristiansen, Sol. Energy Mater. Sol. Cells, 2005, 89, 275-285.

10 A. Du, B. Zhou, Z. Zhang and J. Shen, Materials, 2013, 6, 941968.

11 Q. Xu, H. Ren, J. Zhu, Y. Bi, Y. Xu and L. Zhang, J. Non-Cryst. Solids, 2017, 469, 14-18.

12 H. Ren, J. Zhu, Y. Bi, Y. Xu and L. Zhang, J. Sol-Gel Sci. Technol., 2016, 80, 635-641.

13 K. Kanamori, M. Aizawa, K. Nakanishi and T. Hanada, Adv. Mater., 2007, 19, 1589-1593.

14 K. Kanamori, M. Aizawa, K. Nakanishi and T. Hanada, J. SolGel Sci. Technol., 2008, 48, 172-181.

15 G. Hayase, K. Kanamori and K. Nakanishi, Microporous Mesoporous Mater., 2012, 158, 247-252.

16 G. Hayase, K. Kugimiya, M. Ogawa, Y. Kodera, K. Kanamori and K. Nakanishi, J. Mater. Chem. A, 2014, 2, 6525-6531.

17 G. Hayase, S. Nagayama, K. Nonomura, K. Kanamori, A. Maeno, H. Kaji and K. Nakanishi, Journal of Asian Ceramic Societies, 2017, 5, 104-108.

18 G. Hayase, K. Kanamori, A. Maeno, H. Kaji and K. Nakanishi, J. Non-Cryst. Solids, 2016, 434, 115-119.

19 G. Zu, T. Shimizu, K. Kanamori, Y. Zhu, A. Maeno, H. Kaji, J. Shen and K. Nakanishi, ACS Nano, 2018, 12, 521-532.

20 D. M. Smith, R. Deshpande and C. Jeffrey Brinke, MRS Proceedings, 1992, 271, 104-112.

21 S. S. Prakash, C. J. Brinker and A. J. Hurd, J. Non-Cryst. Solids, 1995, 190, 264-275.

22 F. Schwertfeger, D. Frank and M. Schmidt, J. Non-Cryst. Solids, 1998, 225, 24-29.

23 G. Liu, B. Zhou, X. Ni, J. Shen, G. Wu, A. Du and G. Zu, J. SolGel Sci. Technol., 2012, 62, 126-133.

24 Y. Li, A. Du, J. Shen, Z. Zhang, G. Wu and B. Zhou, J. Porous Mater., 2017, DOI: 10.1007/s10934-017-0533-8.

25 C. J. Brinker and G. W. Scherer, Sol-gel science: the physics and chemistry of sol-gel processing, Academic press, 2013.
26 S. Wu, A. Du, Y. Xiang, M. Liu, T. Li, J. Shen, Z. Zhang, C. Li and B. Zhou, RSC Adv., 2016, 6.

27 L.-J. Wang, S.-Y. Zhao and M. Yang, Mater. Chem. Phys., 2009, 113, 485-490.

28 A. V. Rao and M. M. Kulkarni, Mater. Res. Bull., 2002, 37, 1667-1677.

29 D. Zhang, B. L. Williams, E. M. Becher, S. B. Shrestha, Z. Nasir, B. J. Lofink, V. H. Santos, H. Patel, X. Peng and L. Sun, Adv. Compos. Hybrid. Mater., 2018, 1, 177-184.

30 L. Dashairya, M. Rout and P. Saha, Adv. Compos. Hybrid. Mater., 2018, 1, 135-148.

31 L. Shao, X. Cheng, Z. Wang, J. Ma and Z. Guo, J. Membr. Sci., 2014, 452, 82-89.

32 H. El Rassy and A. C. Pierre, J. Non-Cryst. Solids, 2005, 351, 1603-1610.

33 R. Joseph, A. Shanmin Zhang and W. T. Ford, Macromolecules, 1996, 29, 1305-1312.

34 J. Lin, H. Chen, T. Fei, C. Liu and J. Zhang, Appl. Surf. Sci., 2013, 273, 776-786.

35 J. Huang, Y. Cao, Q. Shao, X. Peng and Z. Guo, Ind. Eng. Chem. Res., 2017, 56.

36 Y. H. Kim, D. K. Lee, H. G. Cha, C. W. Kim and Y. S. Kang, J. Phys. Chem. C, 2007, 111, 3629-3635.

37 G. Liu, B. Zhou, A. Du, J. Shen and G. Wu, Colloids Surf., A, 2013, 436, 763-774.

38 T. Li, B. Zhou, A. Du, Y. Xiang, S. Wu, M. Liu, W. Ding, J. Shen and Z. Zhang, J. Sol-Gel Sci. Technol., 2017, 84, 96103.

39 A. C. Pierre, a. E. Elaloui and G. M. Pajonk, Langmuir, 1998, 14, 66-73.

40 A. Emmerling, R. Petricevic, A. Beck, P. Wang, H. Scheller and J. Fricke, J. Non-Cryst. Solids, 1995, 185, 240-248.

41 G. M. Pajonk, J. Non-Cryst. Solids, 1998, 225, 307-314.

42 C. Li, X. Cheng, Z. Li, Y. Pan, Y. Huang and L. Gong, J. NonCryst. Solids, 2017, 457, 52-59. 